\title{
Optical coherence tomography guided thrombectomy through a "home-made" aspiration catheter in a young patient with myocardial infarction. To stent or not to stent?
}

\author{
Andreas S. Triantafyllis ${ }^{1,2}$, Aimilianos N. Kalogeris ${ }^{2}$, Joost D.E. Haeck ${ }^{3}$, Koen Teeuwen ${ }^{1}$, Athanasios J. Manolis ${ }^{2}$ \\ ${ }^{1}$ Department of Cardiology, Catharina Ziekenhuis Eindhoven, the Netherlands \\ ${ }^{2}$ Department of Cardiology, Asklepeion General Hospital, Athens, Greece \\ ${ }^{3}$ Department of Cardiology, Medisch Centrum Leeuwarden, the Netherlands
}

Adv Interv Cardiol 2020; 16, 4 (62): 486-490

DOI: https://doi.org/10.5114/aic.2020.101777

\section{Introduction}

Invasive coronary angiography (CA) is the traditional imaging modality for evaluation of the coronaries and guidance of percutaneous coronary intervention (PCI) during treatment of ST-segment elevation myocardial infarction (STEMI) [1]. However, CA provides just a two-dimensional luminography of the coronaries, without additional insights into plaque morphology, extent of atherosclerosis, thrombus burden and mechanisms of vessel occlusion [2].

The use of optical coherence tomography (OCT) delivers high-resolution images that accurately depict vessel and lesion characteristics, including the presence of thrombus, provides incremental assistance in stent implantation and minimizes stent-related issues [2]. OCT may also reveal the pathophysiology of arterial thrombosis, and optimize therapeutic options during $\mathrm{PCl}$, especially in young patients where mechanisms of STEMI may vary [3].

Though plaque rupture remains the most common etiology of myocardial infarction (MI), in patients younger than 40-45 years unique syndromes such as plaque erosion, coronary microvascular dysfunction, spontaneous coronary artery dissection (SCAD), myocarditis, coronary embolism, hypercoagulable state and coronary spasm (drug related or not) are prevalent [3]. Consequently, intracoronary imaging is essential to clarify the underlying mechanisms when treating young $\mathrm{MI}$ patients, since stenting might not be the holy grail in this subgroup. Thrombus aspiration, though not routinely advocated by the guidelines, is required in selected patients for the recovery of coronary flow during primary $\mathrm{PCI}[1]$.
Previous reports have highlighted that in selected young patients presenting with STEMI, lone thrombus aspiration without balloon angioplasty or stenting is feasible and is associated with favorable short and long-term outcomes [4]. Dedicated thrombectomy catheters have been developed, but one size does not fit all anatomies, with some catheters being too small to accommodate a large thrombotic burden [5].

\section{Aim}

We report the case of a young patient who presented with anterior STEMI and underwent thrombectomy of a large free-floating thrombus through a "home-made" aspiration device, assembled from a guiding extension catheter and a guide catheter. OCT showed no signs of plaque rupture, allowing deferral of stent implantation.

\section{Case report}

A 38-year-old man, with positive family history for coronary artery disease, presented with acute chest pain. Electrocardiography revealed hyperacute $T$ waves in leads I, aVL, V2- > V6, corresponding with the diagnosis of an anterolateral STEMI (Figure $1 \mathrm{~A}$ ). He was loaded with $250 \mathrm{mg}$ of aspirin, $180 \mathrm{mg}$ of ticagrelor and underwent emergency $C A$, via the right radial artery approach. CA revealed a subtotal occlusion of the proximal segment of the left anterior descending artery (LAD) with the presence of a mobile thrombus (Figure $1 \mathrm{~B}$ arrow) whereas the left main stem, circumflex and right coronary artery were normal. In view of the free-floating thrombus in the proximal segment of the LAD we decided to proceed with thrombus aspiration. After administration of 7500 IU of

Corresponding author:

Andreas S. Triantafyllis MD, PhD, Catharina Ziekenhuis Eindhoven, the Netherlands, e-mail: andtridoc@yahoo.gr Received: 24.08.2020, accepted: 15.10.2020. 

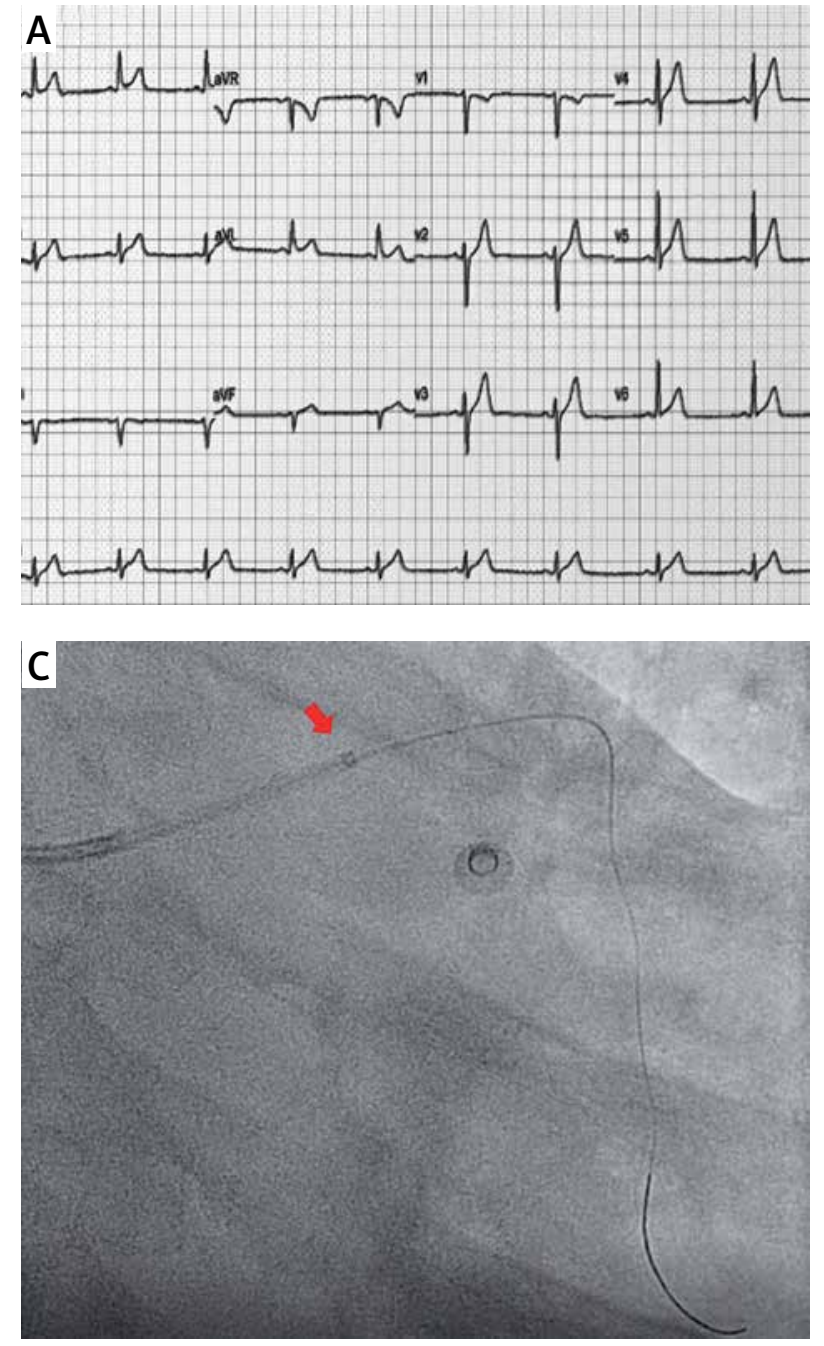

unfractionated heparin via a 6-French 3.5 extra back-up (EBU, Medtronic) guiding catheter, we tried to perform thrombosuction through a standard 6-French aspiration catheter, without success. We hypothesized that the small inner diameter $(1.1 \mathrm{~mm})$ of the standard aspiration catheter (Medtronic, Export) was not large enough to aspirate the large thrombus burden accumulated in the LAD.

Taking into consideration the large diameter of the vessel at the lesion site (between 3.5 and $4.00 \mathrm{~mm}$ ), the proximal location of the thrombus and the large thrombus burden, we decided to construct a wider diameter "home-made" aspiration device by using a 6-French guiding extension catheter, which has an inner diameter of $1.45 \mathrm{~mm}$ and would theoretically offer a greater aspiration potential. Specifically, a 6-French Guidezilla II guide extension catheter (Boston Scientific) was advanced through the EBU guiding catheter at the proximal LAD (Figure $1 \mathrm{C}$, arrow). Successful thrombectomy was performed through the "guide extension-guiding catheter" assembly via a 20 cc luer-lock vacuum aspiration sy-

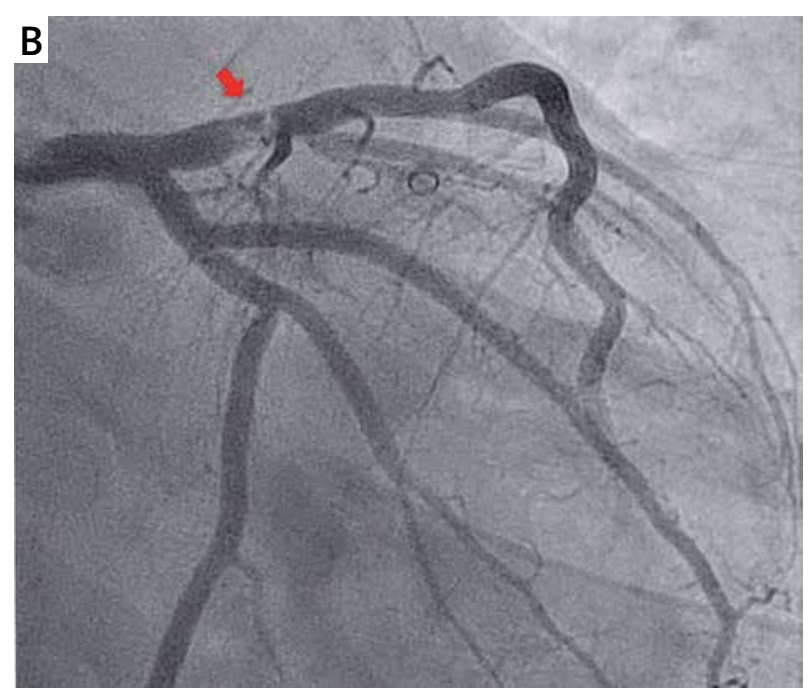

Figure 1. A - ECG showing anterolateral STEMI. B - Angiography revealed the presence of thrombus (arrow) in the proximal LAD. C - Guide extension catheter (arrow) through the guiding catheter at the proximal LAD

ringe attached to the manifold by a three-way stopcock (Figure $2 \mathrm{~A}$ ). Prior to any further manipulation, vigorous back-bleeding through the Y-connector was performed, to eliminate any chance of air or thrombus embolism. Intracoronary injection of tirofiban followed, to ensure adequate resolution of thrombus microparticles, resulting in an excellent angiographic result (Figures $2 \mathrm{~B}, \mathrm{C}$ ). OCT illustrated normal lumen area with presence of minimal thrombus remnants (Figures 2 D, E, arrow), intima thickening and fibroatheroma (Figure $2 \mathrm{D}$, asterisk) without any signs of plaque rupture at the lesion site, allowing deferral of stent implantation. Control CA after 3 days revealed a perfect result.

The patient was discharged in good condition with lifelong aspirin and ticagrelor for 1 year. At 3-month follow-up he was asymptomatic with a normal echocardiogram. Further investigation excluded paradoxical emboli but revealed a positive lupus anticoagulant, for which rheumatologists advised lifelong use of aspirin. 

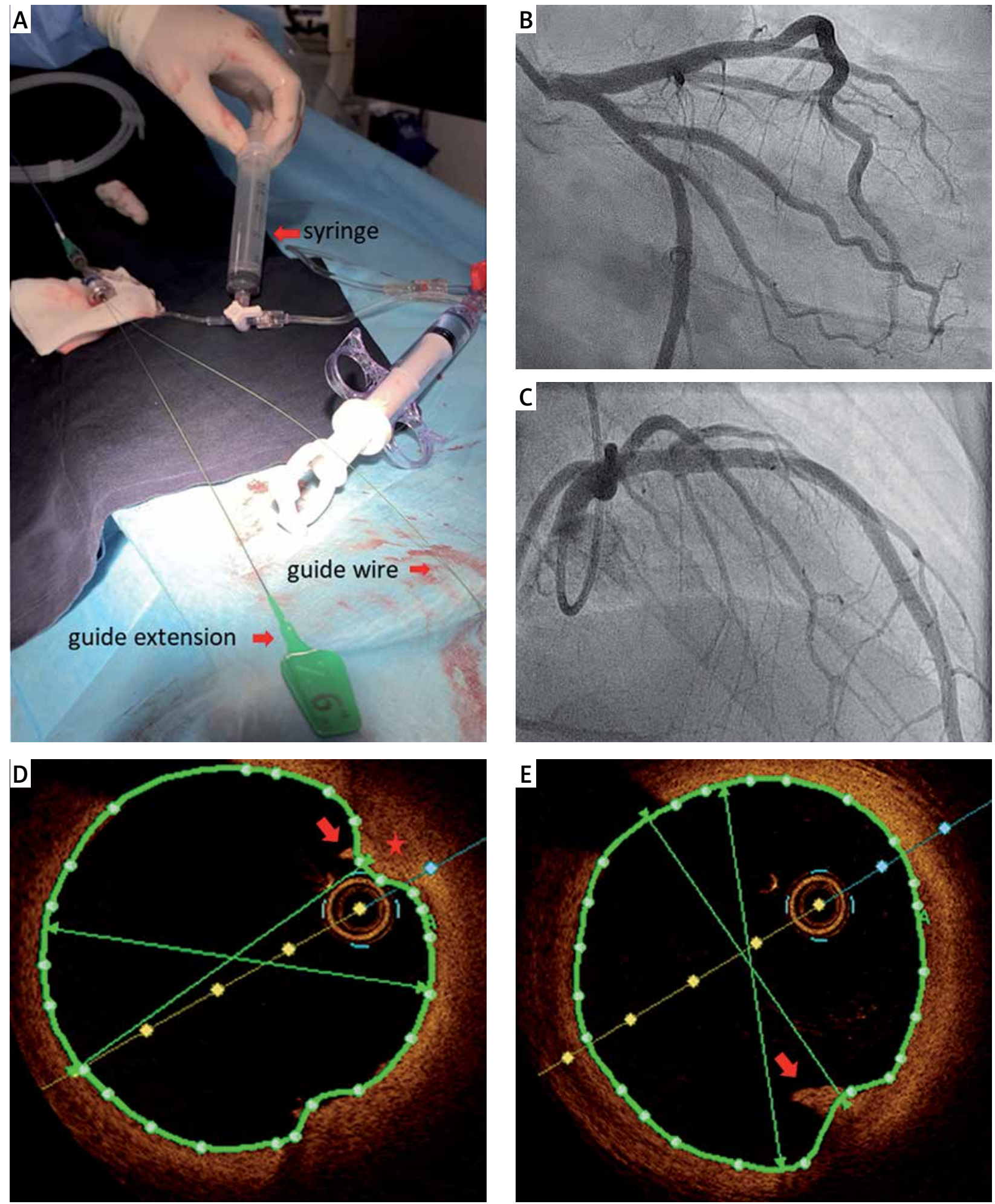

Figure 2. A - Guide extension-guiding catheter assembly: arrows indicate the luer-lock syringe attached to manifold, guidewire and guide extension catheter. B, C - Perfect angiographic result after thrombus aspiration. D, E - OCT illustrating presence of minimal thrombus remnants (D, E arrow), intima thickening and fibroatheroma (D, asterisk) without any signs of plaque rupture 


\section{Discussion}

Restoring coronary blood flow is of utmost importance in the treatment of STEMI during primary $\mathrm{PCl}$. Impaired flow and decreased coronary perfusion is related to reperfusion injury, causing arrhythmias, suboptimal microvascular circulation, contractile abnormalities, permanent myocardial dysfunction and fatal events $[6,7]$. Thrombus aspiration has been proposed as an adjunct to primary $\mathrm{PCl}$ to further improve epicardial and myocardial perfusion by the prevention of distal embolization of thrombotic material and plaque debris [1]. Despite the fact that thrombus aspiration can improve coronary blood flow and resolve ST-segment elevation, it is not routinely advocated by the guidelines (Class IIIA) [1].

The two landmark TASTE and TOTAL trials, which investigated the role of routine manual thrombus aspiration versus conventional $\mathrm{PCl}$, showed no benefit in clinical outcomes of routine aspiration overall or in the high thrombotic risk subgroup [8-10]. Additionally, increased risk of stroke was noted in the TOTAL trial $[9,10]$. Similarly, in a meta-analysis of 17 trials, aspiration thrombectomy was not shown to be of benefit in reducing the risk of death or reinfarction [11]. In the high-thrombus burden subgroup, the trend towards reduced cardiovascular death and increased stroke/transient ischemic at- tack (TIA) calls for further research and use of improved thrombus aspiration technologies in this high-risk subgroup [12].

However, in patients with heavy thrombotic burden, thrombus aspiration should be considered. Several randomized trials have demonstrated the effectiveness and safety of manual thrombus aspiration during primary $\mathrm{PCl}$. The majority of studies in the literature conclude that thrombectomy improves TIMI flow and provides rapid resolution of ST segment deviation. The TAPAS and EXPIRA trials, along with a few meta-analyses, reported that thrombectomy improves the long-term clinical outcome of reducing cardiac death and major adverse cardiovascular events (MACE). However, these studies showed no statistical significance for the prediction of MACE $[13,14]$. The EXPIRA trial illustrated a reduction of the infarct area in cardiac magnetic resonance imaging, after thrombectomy [14]. A survival benefit among patients with STEMI was suggested by the TAPAS trial [13].

In addition, STEMI is an uncommon entity in young adults and its incidence depends on the cut-off age used [15]. Most studies have used an age cut-off of 40-45 years to define young patients with STEMI [3]. It has been reported that $<1 \%$ of patients with STEMI are $\leq 35$ years [15]. Young STEMI patients differ from older patients in

Table I. Characteristics of commonly used thrombus aspiration (A) and guide extension catheters (B)

A. Thrombus aspiration catheters (size $6 \mathrm{Fr}$ )

\begin{tabular}{|c|c|c|c|}
\hline Manufacturer & Device trade name & Catheter length $[\mathrm{cm}]$ & $\begin{array}{l}\text { Aspiration lumen area/diameter } \\
{\left[\left[\mathrm{mm}^{2} / \mathrm{mm}\right]\right.}\end{array}$ \\
\hline Vascular Solutions & Pronto V3 & 140 & $0.93 / 1.09$ \\
\hline \multirow[t]{2}{*}{ Medtronic } & Export AP & 140 & $1.08 / 1.17$ \\
\hline & Diver CE & 145 & $1.01 / 1.14$ \\
\hline Boston Scientific & Fetch 2 & 135 & $0.79 / 1.00$ \\
\hline \multirow[t]{2}{*}{ Terumo } & Eliminate & 140 & $0.79 / 1.00$ \\
\hline & Priority One & 140 & $0.85 / 1.04$ \\
\hline Merit Medical & ASAP AC Kit & 140 & $1.24 / 1.26$ \\
\hline Meril & Aspiron & 140 & $0.69 / 0.94$ \\
\hline Stentys & Stentys AC & 145 & $0.95 / 1.10$ \\
\hline
\end{tabular}

B. Guide extension catheters (size $6 \mathrm{Fr}$ )

\begin{tabular}{lccr} 
Boston Scientific & Guidezilla & 145 & $1.65 / 1.45$ \\
\cline { 2 - 3 } & Guidezilla II & 150 & $1.65 / 1.45$ \\
\hline Vascular Solutions & GuideLiner & 150 & $1.58 / 1.42$ \\
\hline IMDS & Guidion & 150 & $1.58 / 1.42$ \\
\hline Boosting Catheter & QX Medical & 150 & $1.65 / 1.45$ \\
\hline Teleflex & Trapliner & 150 & $1.58 / 1.42$
\end{tabular}


the risk factor profile and in the extent of atheromatic plaque coronary burden. In particular, young coronary patients are characterized by a higher proportion of heavy smoking, a lower proportion of hypertension and diabetes mellitus and a relatively high proportion (15-20\%) of angiographically "normal" coronary arteries [15]. Apart from the classical mechanism of plaque rupture, $\mathrm{MI}$ in young individuals can be attributed to plaque erosion, coronary microvascular dysfunction, SCAD, myocarditis, coronary embolism, hypercoagulable state and coronary spasm related or not to drug use (e.g. cocaine) [3]. In particular, the presence of antiphospholipid antibodies, such as the positive lupus anticoagulant detected in our patient, is a known risk factor for arterial and vein thrombosis by causing a hypercoagulable state [3]. Therefore, diverse underlying mechanisms may be present in young patients with $\mathrm{MI}$, requiring tailored interventional and drug therapy. The use of OCT is essential to reveal the underlying pathophysiology, and optimize therapeutic options [2]. In our patient OCT showed no signs of plaque rupture, allowing deferral of stent implantation after adequate thrombus aspiration.

From a practical point of view, coronary diameter varies between individuals, and commercially available thrombectomy catheters may not be suitable for certain anatomies to achieve sufficient thrombus aspiration (Table I A). Guiding extension catheters have a larger inner diameter and can offer a greater aspiration potential (Table I B). The assembly and use of a home-made aspiration catheter has already been described above. Of note, this technique should only be used if the coronary artery is large enough to accommodate the assembly, since the guide extension catheter can dissect the coronaries and only when the guiding catheter has perfectly engaged the coronary artery to avoid dislocation of thrombus to the systematic circulation during manipulations.

\section{Conclusions}

Diverse underlying mechanisms may be present in young patients with $\mathrm{Ml}$, requiring a case-by-case approach. The use of intracoronary imaging is essential to elucidate the diagnosis and optimize therapeutic options. In selected young patients with MI, OCT-guided lone thrombus aspiration without balloon angioplasty or stenting, either by conventional means or home-made aspiration catheters, can be a feasible and effective therapeutic approach.

\section{Conflict of interest}

The authors declare no conflict of interest.

\section{References}

1. Ibanez B, James S, Agewall S, et al. 2017 ESC Guidelines for the management of acute myocardial infarction in patients presenting with ST-segment elevation. Eur Heart J 2018; 39: 119-77.
2. Räber L, Mintz GS, Koskinas KC, et al. Clinical use of intracoronary imaging. Part 1: guidance and optimization of coronary interventions. An expert consensus document of the European Association of Percutaneous Cardiovascular Interventions. EuroIntervention 2018; 14: 656-77.

3. Gulati R, Behfar A, Narula J, et al. Acute myocardial infarction in young individuals. Mayo Clin Proc 2020; 95: 136-56.

4. Jamil G, Jamil M, Abbas A, et al. "Lone aspiration thrombectomy" without stenting in young patients with ST elevation myocardial infarction. Am J Cardiovasc Dis 2013; 3: 71-8.

5. Ciecwierz D, Mielczarek M, Jaguszewski $M$, et al. The first reported aspiration thrombectomy with a guide extension mother-and-child catheter in ST elevation myocardial infarction due to bacterial vegetation coronary artery embolism. Adv Interv Cardiol 2016; 12: 70-2.

6. Moens AL, Claeys MJ, Timmermans JP, Vrints CJ. Myocardial ischemia/reperfusion-injury, a clinical view on a complex pathophysiological process. Int J Cardiol 2005; 100: 179-90.

7. Resnic FS, Wainstein M, Lee MKY, et al. No-reflow is an independent predictor of death and myocardial infarction after percutaneous coronary intervention. Am Heart J 2003; 145: 42-6.

8. Lagerqvist B, Frobert O, Olivecrona GK, et al. Outcomes 1 year after thrombus aspiration for myocardial infarction. $N$ Engl I Med 2014; 371: 1111-20.

9. Jolly SS, Cairns JA, Yusuf S, et al.; TOTAL Investigators. Outcomes after thrombus aspiration for ST elevation myocardial infarction: 1-year follow-up of the prospective randomised TOTAL trial. Lancet 2016; 387: 127-35.

10. Jolly SS, Cairns JA, Yusuf S, et al.; TOTAL Investigators. Stroke in the TOTAL trial: a randomized trial of routine thrombectomy vs. percutaneous coronary intervention alone in ST elevation myocardial infarction. Eur Heart J 2015; 36: 2364-72.

11. Elgendy IY, Huo T, Bhatt DL, Bavry AA. Is aspiration thrombectomy beneficial in patients undergoing primary percutaneous coronary intervention? Meta-analysis of randomized trials. Circ Cardiovasc Interv 2015; 8: e002258.

12. Jolly SS, James S, Dzavik V, et al. Thrombus aspiration in ST-segment-elevation myocardial infarction: an individual patient meta-analysis. Thrombectomy Trialists Collaboration. Circulation 2017; 135: 143-52.

13. Vlaar PJ, Svilaas T, van der Horst IC, et al. Cardiac death and reinfarction after 1 year in the Thrombus Aspiration during Percutaneous coronary intervention in Acute myocardial infarction Study (TAPAS): a 1-year follow-up study. Lancet 2008; 371 : 1915-20.

14. Sardella G, Mancone M, Canali E, et al. Impact of thrombectomy with EXPort Catheter in Infarct-Related Artery during Primary Percutaneous Coronary Intervention (EXPIRA Trial) on cardiac death. Am J Cardiol 2010; 106: 624-9.

15. Rallidis LS, Triantafyllis AS, Sakadakis EA, et al. Circadian pattern of symptoms onset in patients $\leq 35$ years presenting with ST-segment elevation acute myocardial infarction. Eur I Intern Med 2015; 26: 607-10. 